\title{
Philosophiques
}

\section{La valeur intrinsèque chez Brentano et Moore}

\section{Thomas Baldwin}

Volume 26, numéro 2, automne 1999

La critique de la raison en Europe centrale

URI : https://id.erudit.org/iderudit/004896ar

DOI : https://doi.org/10.7202/004896ar

Aller au sommaire du numéro

\section{Éditeur(s)}

Société de philosophie du Québec

ISSN

0316-2923 (imprimé)

1492-1391 (numérique)

Découvrir la revue

\section{Citer cet article}

Baldwin, T. (1999). La valeur intrinsèque chez Brentano et Moore. Philosophiques, 26(2), 231-243. https://doi.org/10.7202/004896ar

\section{Résumé de l'article}

Dans Principia Ethica, Moore exprime son admiration pour les travaux de Brentano en éthique. Une comparaison entre Moore et Brentano révèle que leurs théories ont effectivement de nombreux points en commun. Mais ils sont en désaccord sur la métaphysique de la valeur intrinsèque. Moore adopte une position réaliste abstraite, alors que Brentano conçoit la valeur intrinsèque en faisant référence à l'" amour correct » : ce qui est bon est ce qui mérite l'amour correct. La position de Brentano présente de nombreux avantages comparativement à celle de Moore, mais elle soulève la question de savoir ce que c'est pour l'amour que d'être " correct ». Brentano s'appuie alors sur notre expérience, comparant notre expérience de certains jugements avec la correction de certains amours. Mais cette position semble aujourd'hui indéfendable. Je suggère de construire une autre analyse de l'" amour correct " qui s'appuie sur certains thèmes caractéristiques de Moore : sa « défense du sens commun » et sa thèse de la « survenance » du moral sur le naturel. Toutefois, je conclus qu'il est difficile de voir comment une telle analyse peut être complétée sans une compréhension de la fiabilité générale de nos sentiments, et qu'une telle analyse semble destinée à nous conduire en direction du naturalisme éthique, déjà refusé par Brentano.
Ce document est protégé par la loi sur le droit d'auteur. L’utilisation des services d’Érudit (y compris la reproduction) est assujettie à sa politique d'utilisation que vous pouvez consulter en ligne.

https://apropos.erudit.org/fr/usagers/politique-dutilisation/ 


\title{
La valeur intrinsèque chez Brentano et Moore
}

\author{
THOMAS BALDWIN \\ Université de York, Royaume-Uni \\ Trb2@york.ac.uk
}

\begin{abstract}
RÉSUMÉ. - Dans Principia Ethica, Moore exprime son admiration pour les travaux de Brentano en éthique. Une comparaison entre Moore et Brentano révèle que leurs théories ont effectivement de nombreux points en commun. Mais ils sont en désaccord sur la métaphysique de la valeur intrinsèque. Moore adopte une position réaliste abstraite, alors que Brentano conçoit la valeur intrinsèque en faisant référence à l'« amour correct » : ce qui est bon est ce qui mérite l'amour correct. La position de Brentano présente de nombreux avantages comparativement à celle de Moore, mais elle soulève la question de savoir ce que c'est pour l'amour que d'être « correct». Brentano s'appuie alors sur notre expérience, comparant notre expérience de certains jugements avec la correction de certains amours. Mais cette position semble aujourd'hui indéfendable. Je suggère de construire une autre analyse de l'«amour correct » qui s'appuie sur certains thèmes caractéristiques de Moore : sa «défense du sens commun » et sa thèse de la « survenance » du moral sur le naturel. Toutefois, je conclus qu'il est difficile de voir comment une telle analyse peut être complétée sans une compréhension de la fiabilité générale de nos sentiments, et qu'une telle analyse semble destinée à nous conduire en direction du naturalisme éthique, déjà refusé par Brentano.
\end{abstract}

ABSTRACT. - In Principia Ethica Moore expresses his great admiration for Brentano's ethical writings, and a comparison between Moore and Brentano reveals that their ethical theories have much in common. But they disagree fundamentally on the metaphysics of intrinsic value. Moore adopts an abstract realist position, whereas Brentano interprets intrinsic value by reference to "correct love" : that which is good is that which merits correct love. Brentano's position has many advantages over that of Moore; but it raises the question as to what it is for love to be "correct". Brentano simply relies on our experience at this point, comparing our experience of the evidence of certain judgements with our experience of the correctness of certain loves. But this no longer seems tenable. I suggest that one might try to construct an alternative account of "correct love" by drawing on other themes from Moore : his "defence of common Sense" and his thesis of the "supervenience" of the moral on the natural ; but I conclude that it is difficult to see how such an alternative account can be completed without some understanding of the general reliability of our natural sentiments, and that such an account seems bound to lead in the direction of the kind of ethical naturalism which Brentano repudiated.

Le jeune G. E. M oore conclut, dans son compte-rendu de la traduction anglaise du livre Von U rsprung sittlicher Erkenntnis de Franz Brentano: "Ce livre nous donne une discussion des principes fondamentaux de l'éthique bien meilleure que toutes celles que je connais. ${ }^{1}$

1. M oore, 1903a, p. 115. 
La même année, M oore lui-même publie son traité d'éthique, Principia $E$ thica ${ }^{2}$. D ans la préface de celivre, il répète son admiration pour I'U rsprung de Brentano. $M$ alheureusement, il n'existeà ma connaissance aucun compterendu ou commentaire de Brentano sur le livre de M oore.

Selon M oore et Brentano, le concept fondamental en éthique est celui de valeur intrinsèque, et tous deux sont d'accord sur plusieurs aspects de la théorie de la valeur intrinsèque. $M$ ais, comme nous le verrons, ils sont en désaccord sur un point fondamental, soit le statut de ce concept. J'arguerai que, sur ce sujet, la position de Brentano est préférable à celle de M oore. Je soutiendrai toutefois que la théorie de Brentano n'est pas acceptable dans tous ses détails, et j'essayerai de construire une position bonifiée par deux modifications quasi mooréennes, l'une donnant un rôle au « sens commun » en éthique, l'autre concernant la « survenance » de l'éthique sur le naturel. Je conclurai néanmoins que, malgré ces modifications, la position brentanienne ne peut éviter un problème insoluble sans abandonner la thèse même de Brentano.

II convient d'insister, en premier lieu, sur quelques positions partagées par $\mathrm{M}$ oore et Brentano. Un premier groupe concernele statut des vérités fondamentales de l'éthique. Ils sont tous deux d'accord pour affirmer que :

i) ces vérités sont sui generis (elles ne sont pas réductibles à la psychologie, la sociologie, la politique etc.) ;

ii) ces vérités ne sont pas analytiques ;

iii) ces vérités sont universelles et nécessaires (ou « apodictiques ») ; de chose.

iv) ces vérités concernent la valeur intrinsèque relative de toute espèce

Un second groupe concerne le contenu de la morale. M oore et Brentano s'entendent pour dire que :

i) la fin idéale de la vie est de produire dans le monde le maximum de bien (« utilitarisme idéal ») ;

ii) il existe une grande variété de biens, c'est-à-dire une variété irréductible d'espèces de choses qui sont intrinsèquement bonnes ;

iii) quelques actes mentaux, ou états de conscience, sont les choses les plus valables; parmi ceux-ci, une conscience correcte d'une conscience valable (ce que M oore appelle l'« amour de l'amour ») possède la plus haute valeur ;

iv) la valeur d'une situation complexe est gouvernée par un « principe d'unité organique» qui affirme que cette valeur n'est pas la somme des valeurs de ses aspects constitutifs.

On pourrait croire que cet accord, tout compte fait assez remarquable, indique que M oore a simplement copié le livre de Brentano. En fait, M oore n'a lu ce livre qu'après avoir terminé son propre livre. Je pense, cependant, que l'on peut dire qu'il existait des circonstances favorables à l'expression de

2. Moore, $1903 b$. 
ces idées post-idéalistes à la fin du $19^{\mathrm{e}}$ siècle, et Brentano a sans doute contribué à ce Z eitgeist (alors que, chez M oore, l'effet de l'œuvre de Sidgwick était prépondérant). Brentano, $M$ oore et plusieurs autres pensaient que, en éthique, il était impératif d'éviter deux dogmes : le dogme du réductionnisme (positiviste ou religieux) et le dogme de l'idéalisme transcendantal. Ils croyaient plutôt que la base d'une vraie éthique repose sur une conception de la valeur intrinsèque sui generis.

La grande différence entre la théorie de $M$ oore et celle de Brentano concerne le statut métaphysique de la val eur intrinsèque. Sel on M oore, la val eur intrinsèque des choses constitue une dimension fondamentale et irréductible de la réalité, alors que selon Brentano, au contraire, elle dépend de certains de nos actes mentaux, nos « amours corrects». Puisque la position de M oore est plus simple et mieux connue que celle de Brentano, je commencerai par faire quelques remarques à propos de la première. Son cœur est constitué de la thèse selon laquelle toute chose, situation, état de choses, etc., possède un degré de valeur intrinsèque rel atif à la valeur de toute autre chose, situation ou état de choses. Cette valeur est une dimension de la réalité comparableaux propriétés physiques, commela masse, sauf qu'elle n'est pas ellemême une propriété physique ou « naturelle ». M oore donne plusieurs arguments à l'appui de cette thèse anti-naturaliste, dont le plus important est certainement que, étant donné que la valeur intrinsèque est une dimension fondamentale de la réalité, si elle était une propriété naturelle, elle serait alors elle-même un constituant fondamental de la « nature intrinsèque » des choses, indépendante des autres constituants de cette nature intrinsèque. $M$ ais cette conclusion est incompatible avec une autre thèse assez plausible de $M$ oore sel on laquelle la valeur intrinsèque relative de deux choses dépend de la nature intrinsèque entière de ces deux choses.

Cette dépendance de la valeur envers la nature intrinsèque explique, selon $\mathrm{M}$ oore, le fait que les vérités fondamentales de l'éthique soient nécessaires, car elle implique une connexion nécessaire entre nature et valeur. Cette connexion, cependant, reste problématique : elle ne peut être analytique, et doit donc constituer une espèce de vérité synthétique a priori. $M$ ais comment ces vérités sont possibles demeure inexpliqué chez $M$ oore et est, à mon avis, inexplicable. II jugerait, par exemple, que toutes les explications kantiennes constituent des violations de sa thèse selon laquelle le concept de valeur intrinsèque est le concept fondamental en éthique. Ainsi, selon $M$ oore, il y a une myriade de vérités synthétiques a priori inexplicables concernant la valeur intrinsèque relative de toute espèce de choses, et cette myriade de vérités constitue le matériel fondamental en éthique.

M oore dirait qu'on ne trouverait problématique cet aspect de sa position que si on l'approchait avec une présomption de métaphysique systématique qu'il rejette. $M$ ais même si on veut ainsi écarter toute question métaphysique sur le synthétique a priori, on devrait être embarrassé par l'épistémologie de cette position. En effet, elle implique que nous possédons une 
capacité de discerner, sans argumentation, cette myriade de vérités synthétiques a priori. $\mathrm{Or}$, il est très difficile de comprendre comment nous pouvons nous attribuer une telle capacité et, qui plus est, une capacité fiable, étant donné l'existence de désaccords profonds sur les questions morales comme, par exemple, le fait de tuer des animaux en vue de la consommation humaine. II est également difficile de comprendre comment les jugements mooréens de valeur intrinsèque peuvent nous fournir des motifs pour l'action. En effet, si ces jugements ne concernent qu'une dimension abstraite des choses, sans rapport essentiel à nos besoins ordinaires, nous devons nous demander pourquoi il nous faut nous intéresser à ces jugements.

Comme je l'ai dit, la grande différence entre Brentano et $M$ oore réside dans le fait que, selon Brentano, le concept de valeur intrinsèque est fondé sur nos sentiments et ne constitue pas une dimension fondamentale de la réalité. Pour cette raison, la position brentanienne évite les difficultés métaphysiques qui affaiblissent celle de $M$ oore. II semble qu'on ait alors une théorie permettant d'éviter les autres problèmes. M ais avant de voir s'il en va bien ainsi, il nous faut préciser la position de Brentano. Lorsquej'ai dit que, selon lui, le concept de valeur intrinsèque était fondé sur nos sentiments, on a pu avoir l'impression que sa position était explicitement subjectiviste. $M$ ais il n'en est rien, car il affirme qu'une chose possède une valeur intrinsèque positive seulement lorsqu'elle est l'objet d'un « amour correct » (L iebe richtig) ou lorsqu'elle mérite un tel amour. Je reviendrai plus tard sur cet acte mental qu'est l'« amour ». Je veux d'abord examiner l'exigence voulant que cet amour soit « correct ». II est clair que le but de cette dernière est d'assurer l'objectivité des jugements de valeur intrinsèque. Ainsi l'impression d'une position explicitement subjectiviste était-elle erronée. La question est de savoir si Brentano peut démontrer d'une manière convaincante comment ce but peut être atteint.

En examinant cette question, il faut toujours garder à l'esprit la primauté, chez Brentano, du phénomène del'amour correct. Dans un bref commentaire sur la position d'A ristote, il écrit :

J'ai dit que la tentation à laquelle succombait Aristote semble facile à comprendre. Elle a sa source dans le fait que lorsque nous avons une émotion positive dont nous éprouvons la correction par expérience, nous acquerrons aussi la connaissance que l'objet de l'émotion est quelque chose qui est bon. II est facile de confondre la relation entre l'émotion et la connaissance. $0 \mathrm{n}$ peut donc supposer, par erreur, que l'amour d'une chose bonne est une conséquence de la connaissance qu'elle est bonne, et que l'amour est vu comme correct parce qu'il est approprié à la connaissance. ${ }^{3}$

Comme l'indique ce passage, Brentano soutient quela perception intérieure, qui nous donne la connaissance de nos actes mentaux, peut aussi nous donner une perception que notre amour est correct ; et cette expérience de la cor-

3. Brentano, 1889, seconde édition, p. 59 ; traduction anglaise, p. 89. 
rection de notre amour doit nous amener à la connaissance que l'objet de l'amour est bon.

$\mathrm{H}$ abituellement, Brentano explique cette thèse en faisant référence au rapport intime entre l'expérience de l'évidence d'un jugement et notre connaissance de sa vérité. Sur cette question du rapport entre l'évidence et la vérité, il écrit : « dans le cas d'un jugement évident, nous avons l'expérience de sa correction. C'est seulement dans la mesure où nous discernons certains jugements que le mot "vrai" a un sens. ${ }^{4}$ » Sans doute suppose-t-il une similitude entre l'expérience de la correction d'une émotion et la signification du mot «bon ». M ais, en réfléchissant sur l'analogie que fait Brentano entre notre expérience de la correction d'une émotion et celle de l'évidence d'un jugement, il faut distinguer sa première position sur le rapport entre l'évidence et la vérité de celle qu'il adoptera plus tard. Selon la première position, l'importance de l'expérience de l'évidence d'un jugement est, au premier chef, épistémologique : Brentano soutient que la chaîne des raisons pour nos croyances est fondée sur des jugements que nous éprouvons comme évidents par expérience. Cette position est compatible avec la théorie de la vérité-correspondance que soutient Brentano à cette époque. Plus tard, cependant, il en vint à penser que cette conception de la vérité-correspondance exigeait une superfluité d'existants, par exemple des possibilités réelles qui joueraient le rôle de correspondants pour la vérité des jugements de possibilité. Ainsi, il en vint à développer une nouvelle position incluant une définition de la vérité en termes d'évidence dans une conception déflationniste de la vérité. II écrit :

Pourrions-nous trouver une autre interprétation pour adaequatio qui rendrait cette thèse acceptable? $M$ a réponse serait que cette thèse nous dit ni plus ni moins que : quelqu'un qui juge qu'une certaine chose existe, ou qu'elle n'existe pas, ou qu'elle est possible [...] juge vraiment pourvu que la chose existe, ou qu'elle n'existe pas, ou qu'elle est possible. [...] En effet, si on parle strictement, toutes ces remarques se réduisent à la position suivante : La vérité appartient au jugement de celui qui juge correctement, c'est-à-dire au jugement de celui qui juge d'une chose de la manière dont celui dont le jugement serait évident jugerait de la même chose. ${ }^{5}$

Cette remarque est embêtante, car la thèse déflationniste de la première partie ne demande pas la définition de la vérité de la seconde. De plus, cette définition soulève des problèmes bien connus, car elle semble impliquer une conception anti-réaliste de la vérité, par exemple dans le cas d'un jugement qui concerne le passé et pour lequel il n'existe maintenant aucune information, ou aucun autre fait, qui puisse établir sa vérité ou sa fausseté. $N$ ous ne voulons pas être obligés de conclure que ce jugement n'est ni vrai ni faux parce qu'il n'existe maintenant aucune possibilité de jugement évident sur le

4. Brentano, 1952, p. 152 (traduction anglaise, p. 131).

5. Brentano, 1930, p. 139 ; (traduction anglaise, p. 121-122). 
contenu de ce jugement. Bien sûr, Brentano lui-même ne tirera pas cette conclusion, parce qu'il pensait que l'existence de Dieu impliquait l'existence d'un être qui peut toujours juger d'une manière évidente à propos de toute question. $M$ ais il me semble un peu extraordinaire d'affirmer cettehypothèse métaphysique et en même temps de rejeter la théorie de la vérité-correspondance à cause de ses implications métaphysiques. II serait assez simple de réinterpréter les « correspondants » de cette théorie en termes de l'hypothèse théologique qu'affirma Brentano. Donc, puisque Brentano prit en aversion les implications ontologiques de la théorie de la vérité-correspondance, il eut été préférable qu'il ajoute simplement une théorie déflationniste de la vérité à sa théorie du rôle épistémologique fondamental du jugement évident.

J'ai examiné la théorie brentanienne de l'évidence et de la vérité parce qu'il la prend comme modèle dans son traitement de l'amour correct et du bien. Conséquemment, on doit se demander si la définition du bien en termes d'amour correct proposée par Brentano a des implications anti-réalistes, qui seraient sans doute involontaires. À première vue, elle a de telles implications, parce que sa thèse semble avoir pour conséquence ques'il y a une chose à propos de laquelle un sentiment correct (soit amour ou haine) n'est pas possible, cette chose n'est ni bonne ni mauvaise. $M$ ais, en fait, je pense que cette impression n'est pas fondée, car lorsque Brentano donne sa définition, il écrit toujours que le bien est ce qui mérite l'amour correct. Cette modification, qu'il n'apporte jamais à sa définition de la vérité, implique que dans le cas de la définition du bien, il n'y a pas de menace anti-réaliste, car l'extension à tout ce qui mérite l'amour correct n'est pas limitée par les possibilités de l'expérience d'amour correct.

Pour cette raison, je crois que nous pouvons écarter l'idée que la définition brentanienne du bien implique une position anti-réaliste, quoi que nous pensions de sa définition de la vérité. Ainsi, l'importance de la comparaison entre le jugement évident et l'amour correct repose principalement sur son rôle épistémologique. La conception brentanienne de l'évidence d'un jugement appartient aux questions normatives sur la justification de nos croyances, et la thèse centrale de Brentano est queles normes épistémiques ne peuvent être fondées que sur nos expériences épistémiques. II constate que chaque essai d'introduction d'un nouveau critère extérieur à nos expériences actuelles échoue, parce quela question de savoir si ce critère est satisfait nous renvoie directement à nos expériences actuelles. $M$ ais Brentano ajoute à ce point, tout compte fait légitime, une thèse beaucoup plus problématique : nous avons cette expérience d'évidence seulement dans les deux cas de « perception interne » (c'est-à-dire de conscience de soi) et de vérités analytiques. Cette position, bien connue dans I'histoire de la philosophie, rend douteuse la légitimité de notre croyance en l'existence du monde extérieur et, à mon avis, il serait préférable d'avoir une autre interprétation de la conception brentanienne del'expérience de l'évidence d'un jugement. Pour cetteraison, je propose une modification de sa position qui interprète la notion 
d'expérience de l'évidence à la lumière de nos conceptions ordinaires del'évidence et de la certitude. Ces conceptions utilisent une notion d'évidence qui n'est pas absolument infaillible, mais qui implique que, dans toutes les circonstances ordinaires, on n'aurait pas de raison de douter. Par exemple, il est évident pour moi en ce moment que je suis dans une pièce avec plusieurs autres personnes, et si j'essayais de tenir cette croyance pour fausse, je penserais seulement que si cette croyance était fausse, je ne saurais quoi croire. Bien sûr, ces expériences et habitudes ordinaires ne sont pas infaillibles et nous avons besoin d'une justification théorique de leur légitimité. $M$ ais notre connaissance du monde et de nous-mêmes nous fournit une justification suffisante de leur fiabilité ; et si cet argument dépend de nos théories dont l'évidence repose sur nos expériences ordinaires, c'est précisément une implication de la conception brentanienne de l'évidence qu'il n'existe pas de point d'appui pour l'évidence à l'extérieur de notre expérience actuelle.

Je vais caractériser cette réinterprétation de la conception de l'expérience de l'évidence du jugement comme une modification mooréenne de la théorie brentanienne de l'évidence, parce que ma référence à nos expériences et habitudes épistémiques ordinaires constitue une sorte de défense du sens commun. $M$ ais il faut immédiatement qualifier cette comparaison avec $M$ oore dans le contexte des questions sur la valeur intrinsèque, parce que M oorelui-même ne donne aucun rôleà notre sens commun moral dans Principia Ethica. Au contraire, il s'en remet aux intuitions directes de la valeur intrinsèque des choses. Comme je l'ai cependant déjà indiqué, s'en remettre à de telles intuitions est une méprise. On peut se demander, donc, s'il est possible d'améliorer la position mooréenne en attribuant un rôle fondamental au sens commun moral dans son éthique. À mon avis, la manière d'effectuer une telle modification de la position mooréenne n'est pas claire étant donné sa conception métaphysique de la valeur intrinsèque, et je n'essayerai pas de développer ces questions ici. J e veux concentrer mon attention sur la position de Brentano et sur la possibilité, et l'importance, d'une modification de sa conception de l'expérience de l'amour correct qui ressemble à la modification quej'ai proposée de sa conception de l'expérience du jugement évident.

Chez Brentano lui-même, la comparaison entre jugement évident et amour correct implique l'existence d'une expérience immédiate et incontestable de la correction de certains de nos amours. Comme je l'ai déjà suggéré dans le contexte de la discussion de sa conception de notre expérience de l'évidence, cette thèse me semble être une erreur : nous n'avons pas une compréhension suffisante de l'origine de nos sentiments pour être assurés d'une telle expérience de leur correction. $M$ ais, comme nous l'avons déjà montré pour sa conception de l'expérience de l'évidence du jugement, la position brentanienne recèle une juste perspective : nos sentiments moraux ne peuvent être légitimés que dans une compréhension de notre existence qui utilise notre expérience de ces sentiments. Cette perspective me semble admettre une interprétation quasi mooréenne selon laquelle, en éthique, nous devons 
nous en remettre à nos sentiments ordinaires, à notre sens commun moral, sans espérer que ce dernier nous apparaîtra incontestable ou source immédiate de vérité morale objective.

Si on adopte cette modification quasi mooréenne de la position brentanienne, il faut se demander comment on peut justifier la possibilité d'un point de vue objectif moral fondé seulement sur nos sentiments moraux, sur nos « amours » et « haines». D ans le langage qu'emploie Brentano, cette question équivaut à celle de savoir comment on peut justifier la description d'amours comme « corrects » ou « incorrects ». Bien sûr, Brentano lui-même dirait que cette question repose sur une méprise, parce que nous devrions nous contenter de l'expérience de la correction de certains de nos amours ou de certaines de nos haines. $M$ ais si on abandonne la foi en une telle expérience incontestable, nous avons besoin d'une autre justification de la correction de certains de nos sentiments, c'est-à-dire de la possibilité d'un point de vue moral objectif fondé sur nos sentiments.

L'explication ordinaire de la description d'un sentiment comme « correct » repose sur la vérité d'une implication cognitive du sentiment. Par exemple, la peur implique que l'objet de la peur est dangereux, et on peut dire qu'une peur est « correcte » lorsque son objet est vraiment dangereux. Ainsi, si on transpose une telle explication au cas d'un amour « correct », on doit supposer qu'il existe une implication cognitive similaire pour l'amour. $M$ ais cette thèse soulève immédiatement une sérieuse difficulté pour n'importe quelle théorie brentanienne de la valeur intrinsèque. L'implication cognitive de l'amour qui se présente immédiatement est que l'objet de l'amour est bon. $M$ ais on ne peut définir la correction d'un amour en termes du bien de son objet si, suivant Brentano, on veut définir le bien en termes de l'objet d'un amour correct. Si on adopte une autre thèse, disons celle voulant qu'un amour est correct lorsque son objet donne du plaisir au sujet de l'amour, on se rend compte que l'on doit accepter que la définition du bien en termes d'amour correct implique une position hédoniste qui est incompatible avec la thèse brentanienne selon laquelle il y a une grande variété de biens. A insi, la thèse voulant qu'une explication de la correction d'un amour repose sur I'hypothèse de la vérité d'une implication cognitive de l'amour est absolument fatale pour la position brentanienne. Une autre explication estelle possible?

Sur ce sujet délicat, je vais interrompre la discussion afin d'introduire quelques remarques sur l'identité du sentiment que Brentano appelle « amour » (L iebe). Pour nous, I' « amour » désigne une grande variété de sentiments, y compris les sentiments qui sont distingués en grec par les noms « agapè », « caris », et « éros », l'amour chrétien du Dieu et, en particulier, la conception romantique de l'amour. $\mathrm{N}$ ous avons donc déjà un problème si nous cherchons une seule norme de correction pour toutes ces espèces d'amour. La dernière conception de l'amour, l'amour romantique, soulève une difficulté particulière, car on penserait normalement que cette espèce 
d'amour n'admet pas les descriptions « correct » et « incorrect ». Pour ces raisons, il me semble préférable de chercher une autre caractérisation du sentiment d'après lequel on va tenter de produire une définition du bien. Un candidat bien connu serait le désir, et on doit remarquer que Brentano lui-même définit le meilleur en termes de préférences correctes. $M$ ais il y a ici une difficulté dans le fait que les désirs sont souvent relatifs au sujet : ce que je désire, jele désire pour moi-même ou pour quelqu'un avec qui j'ai un rapport intime (mes enfants, etc.). Ce fait est incompatible avec la pensée que, lorsque ces désirs sont corrects (quoi que cela veuille dire), leur objet est intrinsèquement bon, parce que, selon Brentano, le terme « intrinsèquement » implique «bon-en-soi » et, dans le cas de ces désirs, on doit seulement dire que leur objet est «bon-pour-moi » ou quelque chose de semblable. Pour éviter ces difficultés, il me semble préférable d'identifier simplement à l'évaluation le sentiment d'après lequel on veut définir le bien. Car nous parlons avec justesse de nos évaluations des choses, et la thèse de Brentano exprimée dans ces termes, c'est-à-dire que le bien est ce qui mérite une évaluation positive, semble incontestable, tandis qu'il reste contestable que cette thèse puisse constituer une vraie explication du bien. Une autre question qui demeure discutable est celle du rapport entre l'évaluation et nos autres sentiments, en particulier nos désirs de deuxième ordre. Je pense que Brentano devrait favoriser la thèse selon laquelle l'évaluation est un sentiment sui generis, mais avec des connexions rationnelles avec le désir, car seule cette position peut justifier sa thèse que les jugements de valeur intrinsèque sont eux-mêmes irréductibles et sui generis. $M$ ais il n'est pas nécessaire de prendreici position sur cette dernière question.

Je reviens donc à la question d'une explication de la correction de nos amours ou, comme je le dirai désormais, de nos évaluations. N ous avons affrontéun problème : toute explication qui compte sur la vérité d'une implication cognitive spécifique, quelle qu'elle soit, de l'évaluation est incompatible avec les hypothèses brentaniennes que le bien est définissable en termes d'évaluation correcte et qu'il y a une variété irréductible de biens. $M$ ais il demeure inacceptable de s'en remettre simplement à une expérience directe brentanienne de la correction d'une évaluation. $\mathrm{N}$ ous avons donc besoin d'une autre justification de la possibilité de l'objectivité de nos évaluations, autrement l'accusation sel on laquelle la position brentanienne nous conduit au subjectivisme ou au relativisme serait irréfutable.

La thèse de la survenance de l'éthique sur la nature peut faire avancer notre projet et mérite donc notreattention. Cette thèse constituela deuxième contribution mooréennequeje propose d'utiliser afin de construire une théorie brentanienne alternative de la valeur intrinsèque, même si cette thèse a un statut problématique dans la théorie mooréenne parce qu'elle dépend, dans ce cadre, de l'hypothèse qu'il existe une myriade de connexions nécessaires entre les espèces de choses naturelles et leur valeur intrinsèque. $M$ ais dans le contexte brentanien, où domine la primauté de nos évaluations, la thèse de 
la survenance peut contribuer à l'explication de la possibilité d'évaluations correctes sans dépendre de l'hypothèse antécédente de ces connexions nécessaires. A u contraire, la thèse de la survenance $a$ ici le statut d'un aspect essentiel de l'évaluation - une sorte de vérité synthétique a priori concernant l'évaluation qu'on pourrait justifier dans une théorie générale de la rationalité pratique.

La survenance del'éthique sur le naturel implique directement une condition pour qu'une évaluation soit correcte : elle exige que nos évaluations soient cohérentes avec nos jugements concernant les propriétés axiologiquement neutres : comme on dit souvent, pas de différence évaluative sans différence dans les propriétés axiologiquement neutres qui expliquent la différence évaluative. La question qui se pose alors est de savoir si cette deuxième contribution mooréenne à une position brentanienne peut fournir une explication générale de la possibilité d'une évaluation correcte. $0 \mathrm{n}$ serait d'abord tenté de penser que cette hypothèse n'est pas plausible, parce que la condition de survenance n'est qu'une espèce de demande généralisée de cohérence, et on pourrait penser que la cohérence seule ne peut suffire à l'objectivité. M ais, sur ce point, nous devons nous rappeler qu'il y a aussi la première contribution mooréenne à cette théorie brentanienne alternative, soit la thèse selon laquelle nos évaluations, et l'expérience que nous en faisons, constituent une sorte de sens commun moral. O n peut formuler autrement cette dernière thèse en affirmant que nos évaluations ordinaires sont prima facie correctes. On peut ainsi arguer que la condition de survenance suffit à raffiner ces évaluations prima facie correctes au point qu'elles atteignent un « équilibre réfléchi » et puissent constituer une perspective morale qui mérite la désignation d'objective.

Ces considérations constituent, selon moi, la meilleure défense d'une théorie brentanienne de la valeur intrinsèque. Toutefois, je dois confesser ne pas la trouver convaincante. Pour ce qui est de l'épistémologie, nous pouvons appuyer la défense mooréenne du sens commun par une épistémologie naturalisée qui démontre comment nos jugements ordinaires sont d'habitude fiables et qui (ce qui est très important), nous donne aussi une théorie de l'erreur, c'est-à-dire une explication des conditions dans lesquelles ils ne sont pas fiables. Ainsi donc, il me semble qu'une position brentanienne qui abandonne la foi naïve en la conception d'une expérience directe de la correction de nos évaluations ne peut éviter le besoin d'une "éthique naturalisée », c'est-à-dire d'une théo rie de la nature humaine qui pourrait confirmer la correction prima facie de la plupart de nos évaluations ordinaires. Une telle théorie de la nature humaine doit démontrer comment les objets de ces évaluations positives contribuent à une vie qu'il faut vivre, à « l'être humain qui s'épanouit ». Elle doit aussi fournir une théorie de l'erreur, c'est-à-dire une explication de l'origine de nos évaluations qui démontre comment certaines sont incorrectes. Cette théorie ne sera sans doute pas axiologiquement neutre, et emploiera des suppositions qui expriment notre sens commun moral. 
$M$ ais ce type de circularité est inévitable ; c'est une implication de la thèse de Brentano, quej'ai déjà soulignée plusieurs fois, selon laquelle il n'existe pas de point d'appui pour nos sentiments moraux à l'extérieur de ces sentiments. Ainsi l'exigence d'une théorie de la nature humaine qui confirmera ces sentiments ne fait-elle pas partie d'une vaine quête d'un tel point d'appui. Elle exprime seulement la demande, raisonnable, d'une perspective réfléchie sur nous-mêmes qui puisse confirmer nos sentiments irréfléchis par une explication de ceux-ci qui appartienne à une compréhension générale de la vie humaine, tandis que cette compréhension même ne peut éviter une part de dépendance vis-à-vis de nos sentiments ordinaires.

II faut maintenant expliquer le problème, fondamental pour le point de vue de Brentano sur une théorie de la nature humaine, de la justification de la possibilité d'une expérience d'évaluation correcte. Brièvement, la difficulté est que ce rôle va introduire une perspective aristotélicienne qui contredit la thèse fondamentale de Brentano selon laquelle on doit définir le bien par référence aux sentiments corrects, et non vice versa (il faut se souvenir ici des remarques de Brentano sur A ristote citées plus haut). Car si la justification de la possibilité d'évaluation correcte dépend d'une théorie qui explique le rapport entre l'objet d'évaluation et les conditions d'une vie qu'il faut vivre, nous pouvons déterminer directement quelles choses possèdent une valeur intrinsèque (pour nous) et à quel degré, sans référence à nos évaluations, qu'elles soient correctes ou non. A u contraire, nous ne pouvons confirmer la rectitude de nos évaluations que par référence à la question de savoir si, selon notre compréhension de la vie humaine, leurs objets sont bons. Sans doute restera-t-il ici deux rôles fondamentaux pour nos évaluations et autres sentiments moraux : ils nous donnent le point d'origine inévitable et insurmontableà toute réflexion critique sur l'éthique, et ils constituent eux-mêmes une partie importante d'une vie réfléchie. N ous aurions alors perdu le rôle que Brentano leur accorde, celui de la détermination de la valeur intrinsèque.

Finalement, voici un bref résumé de mon argument. J'ai discuté dequatre positions quant à la possibilité d'une théorie acceptable de la valeur intrinsèque. J'ai d'abord présenté la position du jeune $M$ oore, voulant que la valeur intrinsèque constitue une dimension fondamentale de la réalité. J'ai affirmé, sans argument détaillé, que cette position était inacceptable à cause des problèmes métaphysiques et épistémologiques qu'elle soulève. J'ai ensuite examiné la thèse de Brentano selon laquelle la valeur intrinsèque des choses est déterminée par nos sentiments corrects, et qui se satisfait de I'hypothèse d'une expérience immédiate de la correction de certains sentiments. Cette position évite les problèmes qui minent celle de $M$ oore. $M$ ais j'ai soutenu ques'en remettre à une expérience immédiate de la correction de certains sentiments n'est pas acceptable et constitue, en l'absence d'autres développements, une sorte de subjectivisme qui peut seulement prétendre à I'objectivisme. J'ai essayé, conséquemment, de construire une position intermédiaire, une sorte d'hybride brentano-mooréen, qui ne dépende pas de 
I'hypothèse d'une expérience immédiate de la correction, mais qui emploie deux thèses quasi mooréennes, celle du sens commun moral et celle dela survenance de l'éthique, dans l'espoir d'articuler une position qui constituerait une défense acceptable de la possibilité d'une évaluation correcte. Cependant, j'ai argué que cet espoir demeurait insatisfait. À la fin demon texte, j'ai introduit la thèse selon laquelle on ne peut justifier la possibilité d'évaluations correctes que dans le contexte d'une plus grande compréhension de la vie humaine et de la place de nos sentiments moraux dans cette vie, c'est-àdire dans une éthique naturalisée. J 'ai suggéré (mais, bien sûr, sans le prouver) que cette position est juste en ce qui concerne la question d'une justification de la possibilité d'évaluation correcte. $M$ ais le prix à payer est que cette approche nous donne une conception de valeur intrinsèque « naturaliste » qui ne dépend pas de nos évaluations, parce qu'elle explique la possibilité qu'une évaluation soit correcte en termes de la valeur de son objet, et non l'inverse.

Donc, le projet brentanien dans I'U rsprung sittlicher E rkenntnis, soit la recherche d'une conception de la valeur intrinsèque fondée sur nos sentiments corrects, échoue devant le choix entre la troisième et la quatrième de ces alternatives. N ous pouvons poser un dilemme à Brentano : soit il admet sa définition de la valeur en termes de nos sentiments, et accepte que son éthique constitue une espèce de subjectivisme éthique qui ne donne pas un sens propreà l'expression « amour correct » (Gibbard, dans son livreW ise Choices, A pt Feelings ${ }^{6}$, nous fournit aujourd'hui un exposé clair et distinct d'une position de ce type), soit, s'il continue d'aspirer à la possibilité que nos sentiments soient corrects et que la connaissance éthique soit objective, il abandonne sa définition de la valeur intrinsèque et la remplace par une théorie aristotélicienne de l'être humain qui s'épanouit. Comme chez M oore, l'aspiration de Brentano à trouver une position intermédiaire entre le naturalisme aristotélicien et toute forme de subjectivisme idéaliste reste irréalisable.

\section{Bibliographie}

Brentano, F., Von Ursprung sittlicher Erkenntnis, Leipzig, Duncker und $\mathrm{H}$ umblot, 1889 (traduction anglaise: Hague, C., The $\mathrm{O}$ rigin of $\mathrm{O}$ ur Knowledge of Right and Wrong, London, Constable, 1902); deuxième édition (Kraus, O., dir.) : Leipzig, Felix M einer, 1922 (traduction anglaise: Chisholm, R. et Schneewind, E., London, R outledge, 1969)

- - , Wahrheit und Evidenz (Kraus, O., dir.), Leipzig, Felix M einer, 1930 (traduction anglaise: Chisholm, R., et al., The True and the Evident, London, Routledge, 1966)

6. Gibbard, 1990. 
- - , G rundlegung und A ufbau der E thik, Bern, A. Franke, 1952 (traduction anglaise : Schneewind, E., The Foundation and Construction of Ethics, London, Routledge, 1973)

Gibbard, A., W ise Choices, A pt Feelings, Oxford, Clarendon Press, 1990

M oore, G.E., Compte-rendu de Brentano, 1889, International Journal of Ethics, 14, 1903a, p. 115-123

_- , Principia Ethica, Cambridge, Cambridge University Press, 1903b 
